\title{
Trapping and cooling a mirror to its quantum mechanical ground state
}

\author{
M. Bhattacharya and P. Meystre \\ Department of Physics, The University of Arizona, Tucson, Arizona 85721
}

(Dated: November 2, 2018)

\begin{abstract}
We propose a technique aimed at cooling a harmonically oscillating mirror to its quantum mechanical ground state starting from room temperature. Our method, which involves the two-sided irradiation of the vibrating mirror inside an optical cavity, combines several advantages over the two-mirror arrangements being used currently. For comparable parameters the three-mirror configuration provides a stiffer trap for the oscillating mirror. Furthermore it prevents bistability from limiting the use of higher laser powers for mirror trapping, and also partially does so for mirror cooling. Lastly, it improves the isolation of the mirror from classical noise so that its dynamics are perturbed mostly by the vacuum fluctuations of the optical fields. These improvements are expected to bring the task of achieving ground state occupation for the mirror closer to completion.

PACS numbers: 42.50.Pq, 42.65.Sf, 85.85.+j, 04.80.Nn
\end{abstract}

The observation of quantum dynamics in truly macroscopic objects is a challenging task that, although not yet achieved, has begun to look more and more feasible [1, 2, 3, 4, 5] as a result of recent experimental advances that include novel cooling techniques, progress in nanofabrication, and the improved control of decoherence. This is an exciting prospect, as it would enable us to explore the quantum-classical boundary [6] as well as to test quantum mechanics in an entirely new regime [7]. The implementation of characteristically quantum mechanical phenomena such as entanglement and superposition at a macroscopic scale [8] has broad implications, for instance in the impending merger of quantum optics with condensed matter physics [9]. It also signals direct technological benefits for areas like quantum measurement [10] and communication [11]. Additional potential applications include the interferometric detection of gravitational waves [12] and atomic force microscopy [13].

A promising route to these objectives seems to be through the use of optomechanical systems, particularly of mirror cavities coupled to laser radiation [14, 15, 16]. Experimentally two-mirror cavities have been used, with one mirror held fixed and another mounted on a spring of frequency $\Omega_{M}$ and damped at a rate $\Gamma_{M}$ [Fig:1(a)]. It has been demonstrated that radiation pressure in the cavity can change these quantities to new values $\Omega_{\text {eff }}$ and $\Gamma_{\text {eff }}$ [17]. To bring the mirror to its quantum mechanical ground state starting from temperature $T$ the number of quanta [17]

$$
n=\frac{k_{B} T}{\hbar \Omega_{M}} \frac{\Gamma_{M}}{\Gamma_{\mathrm{eff}}}\left(\frac{\Omega_{M}}{\Omega_{\mathrm{eff}}}\right)^{3},
$$

have to be reduced to $<1$. Here $k_{B}$ and $\hbar$ are Boltzmann's and Planck's constants.

Most progress so far has been achieved by using the technique of cold damping where the thermal Brownian motion heating up the oscillator is countered by the effect of laser radiation, which increases the mirror damping while introducing very little noise [18]. As a result the ratio $\Gamma_{M} / \Gamma_{\text {eff }}$ can be decreased to about $10^{-4}$ either passively or by using active feedback cooling. This yields $n \sim 10^{4}$ for typical parameters even if the base temperature is cryogenically reduced to $T \sim 4 \mathrm{~K}$ [19]. We mention here that $n \sim 25$ has also been attained by coupling nanomechanical resonators to single-electron transistors in the solid state [5]. Cold damping works best for oscillating mirrors with high mechanical frequencies and quality factors and high-finesse cavities. However mirror heating effects due to absorption [1] and the onset of bistability [14] impose a limit to the laser powers that can be used. Improving the cavity finesse may reduce mirror heating, but it also lowers the power threshold for bistability. We also note that cold damping works for laser fields tuned below the cavity resonance [1]. For the conditions just described there is only a small downshift of the mechanical resonance frequency, i.e. $\Omega_{M} / \Omega_{\text {eff }} \sim 1$.

On the other hand, Eq.(1) indicates that if the spring could be made stiffer [20, 21, 22, 23] even by an order of magnitude, the number of quanta could be lowered by three orders of magnitude. However this requires the radiation to be tuned above the cavity resonance, which precludes cold damping. The resolution to this problem has been demonstrated very recently [24] in an experiment that demonstrated the almost independent control of $\Gamma_{\text {eff }}$ and $\Omega_{\text {eff }}$ with the use of two radiation fields of different powers and detunings. One laser field was used to stiffen the spring while the other achieved cold damping, leading to $n \sim 10^{3}$ starting from room temperature. This method also makes it possible to work with weak mechanical attachment of mirrors (small $\Omega_{M}$ ), implying a lower coupling to thermal noise and longer thermal decoherence times. Forming tighter traps therefore seems to be a way to surmount the current limitations of cold damping in reaching the mirror ground state.

Accounting for these observations, the present Letter proposes and analyzes a mirror cooling and trapping configuration that provides stiffer traps, largely removes the problem of bistability and better isolates the mirror from thermal noise. These improvements relax considerably the difficulties in cooling the macroscopic mirror to its quantum mechanical ground state. The arrangement that we propose involves placing a mirror with two per- 

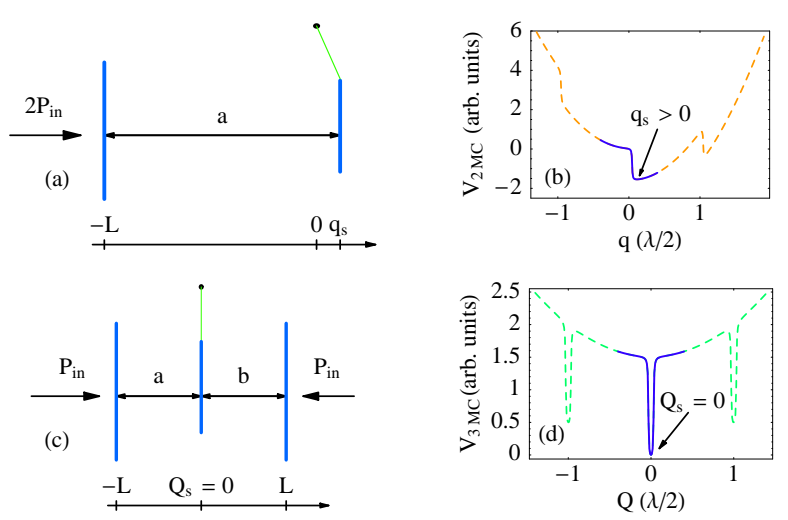

FIG. 1: (Color online).(a) Two-mirror cavity (2MC), with $q_{s}$ the static recoil due to radiation pressure. (b) The corresponding mirror trapping potential in the static limit. The dashed line shows the full classical solution, while the solid line is the $\omega=0$ limit of the single-mode quantum theory. (c) Three-mirror cavity (3MC). (d) The corresponding mirror trapping potential in the static limit. The dashed line shows the full classical solution, while the solid line is the $\omega=0$ limit of the two-mode quantum theory. The parameters labelling the figures are defined in the text.

fectly reflecting surfaces inside a cavity where the other mirrors are transmissive but not movable [Fig[1(c)]. The cavity is then driven by two different laser fields, one for cooling and the other for trapping from both sides. The three-mirror cavity (3MC) configuration leads to potential wells for the movable mirror that are tighter and more harmonic than is the case for the two-mirror cavity (2MC) as illustrated in Fig 1. Such a geometry was proposed many years ago and shown to provide superior mirror confinement, in addition to being more stable than the $2 \mathrm{MC}$ when subjected to Brownian noise [20]. However the treatment underlying that proposal was classical, constructed using solutions to the classical Maxwell's equations for the optical fields in the Fabry-Pérot, the trap frequencies were derived in the static limit, and the noise analysis did not account for fluctuations in the laser fields.

Here we show that tight confinement of the movable mirror in the $3 \mathrm{MC}$ is retained in a dynamical quantum mechanical picture that fully accounts for damping and noise. As expected the classical results previously obtained are regained from the quantum treatment in the appropriate limit [see Figs 1(b) and (d)]. The key point is then that the higher symmetry of the 3MC allows us to significantly improve the trap stiffness and to partially remove the bistability that is a dominant feature of the $2 \mathrm{MC}$ configuration. These conclusions follow from the fact that in the $3 \mathrm{MC}$ the movable mirror does not possess the static recoil due to radiation pressure that follows from the lack of symmetry in the 2MC.

We now proceed to outline a derivation of our results for the 3MC, and contrast them to a similar treatment of the $2 \mathrm{MC}$ for comparison. For our purposes it is adequate to consider external pumping fields at a single frequency, although in practice two frequencies have to be used as discussed above. We first consider cavities without control and subsequently include electronic feedback in our discussion.

The Hamiltonian for the 3MC shown in Fig प(c) can be written as [8]

$H=\hbar \omega_{c}\left(a^{\dagger} a+b^{\dagger} b\right)+\frac{P^{2}}{2 M}+\frac{1}{2} M \Omega_{m}^{2} Q^{2}-\hbar \xi\left(a^{\dagger} a-b^{\dagger} b\right) Q$,

where $a$ and $b\left(a^{\dagger}\right.$ and $\left.b^{\dagger}\right)$ are the bosonic annihilation (creation) operators for the electromagnetic modes of equal frequency $\omega_{c}$ in the left and right sub-cavities of length $L, \xi=\omega_{c} / L$ is the optomechanical coupling constant and $P, Q$ are the momentum and displacement operators of the middle mirror of mass $M$. It is assumed in the following that $Q \ll \lambda$, the optical wavelength.

The first term in the Hamiltonian corresponds to the energy of the optical fields in the two sub-cavities, the next two terms account for the energy of the oscillating middle mirror and the last term describes the effect of radiation pressure on that mirror. We include damping and noise in our treatment via the familiar input-output theory. The Hamiltonian (2) yields then the quantum Langevin equations 25]

$$
\begin{aligned}
& \dot{a}=-\left[i(\Delta-\xi Q)+\frac{\gamma}{2}\right] a+\sqrt{\gamma} a^{\text {in }}, \\
& \dot{b}=-\left[i(\Delta+\xi Q)+\frac{\gamma}{2}\right] b+\sqrt{\gamma} b^{\text {in }}, \\
& \dot{Q}=P / M, \\
& \dot{P}=-M \Omega_{M}^{2} Q+\hbar \xi\left(a^{\dagger} a-b^{\dagger} b\right)-\frac{\Gamma_{M}}{2 M} P+\epsilon^{\text {in }},
\end{aligned}
$$

where $\gamma$ is the decay rate of both the left and right subcavities, and $\Delta=\omega_{c}-\omega_{L}$ is the detuning introduced to account for the difference between the cavity resonance and the laser frequency $\omega_{L}$. The operators $a^{\text {in }}$ and $b^{\text {in }}$ are noise operators describing the fields incident on the cavity. Their mean values $\left\langle a^{\text {in }}(t)\right\rangle=a_{s}^{\text {in }},\left\langle b^{\text {in }}(t)\right\rangle=$ $b_{s}^{\text {in }}$ describe classical fields pumping the cavities, and their fluctuations are assumed to be delta-correlated $\left\langle\delta a^{\text {in }}(t) \delta a^{\text {in }, \dagger}\left(t^{\prime}\right)\right\rangle=\left\langle\delta b^{\text {in }}(t) \delta b^{\text {in }, \dagger}\left(t^{\prime}\right)\right\rangle=\delta\left(t-t^{\prime}\right)$, and add vacuum noise to the cavity modes. Finally, the operator $\epsilon^{\text {in }}$ describes the Brownian noise resulting from the coupling of the middle mirror to the environment. Its mean value is zero, and its fluctuations at temperature $T$ are characterized by the correlation $\left\langle\delta \epsilon^{\mathrm{in}}(t) \delta \epsilon^{\mathrm{in}}\left(t^{\prime}\right)\right\rangle=$ $\frac{\Gamma_{M}}{2 M} \int_{-\infty}^{\infty} \frac{d \omega}{2 \pi} e^{-i \omega\left(t-t^{\prime}\right)} \hbar \omega\left[1+\operatorname{coth}\left(\frac{\hbar \omega}{2 k_{B} T}\right)\right][25]$.

The steady-state values of the dynamical variables can be found by setting the time-derivatives in Eq.(3) to zero. This yields

$$
a_{s}=b_{s} \equiv f_{s}=\frac{\sqrt{\gamma} f_{s}^{\text {in }}}{\left(\frac{\gamma^{2}}{4}+\Delta^{2}\right)^{1 / 2}}, \quad Q_{s}=0, \quad P_{s}=0,
$$

where $f_{s}^{\text {in }}=\left|a_{s}^{\text {in }}\right|=\left|b_{s}^{\text {in }}\right|$ is the amplitude of both pumping fields. The phases of these fields have been independently chosen such that $f_{s}$ is real. This can be done 
without loss of generality as our model is insensitive to the mutual (in)coherence of the two pumping fields. For later use we define the input power from either side as $P_{\text {in }}=\hbar \omega_{c}\left|f_{s}^{\text {in }}\right|^{2}$.

It can be shown from the steady-state equations that for $\Delta<0$, which corresponds to trapping and heating of the middle mirror, that $Q_{s}=0$ is always the only real solution and hence there is no multistability in the position of the mirror. This is in contrast to the $2 \mathrm{MC}$ where bistability is expected for $|\Delta| \geqslant \sqrt{3} \gamma / 2$ [26], and has been observed [20]. Physically, this is a result of the fact that the cavity length changes with $P_{i n}$ in a fashion mathematically similar to the effect of a Kerr medium in conventional bistability. The implication for mirror control is that higher laser powers can be used in the $3 \mathrm{MC}$ than in the $2 \mathrm{MC}$ to strengthen the trapping effects of radiation pressure, and that these powers are now limited only by absorption heating effects.

The stiffening due to the trapping laser field also improves the situation with regard to the bistability that occurs due to the cooling (and anti-trapping) laser field at $\Delta>0$ : It takes more power to make a stiff mirror bistable, hence the trapping field raises the power threshold for the onset of bistability due to the cooling field. We show below that the $3 \mathrm{MC}$ can provide stiffer traps than the 2MC. This implies that higher laser power can be used for cooling in the $3 \mathrm{MC}$ than in the $2 \mathrm{MC}$.

We mention here that depending on the laser powers used for cooling and trapping fields, their respective detunings have to be chosen to satisfy requirements of dynamic stability and are not completely independent [24]. Typically, for the relatively high powers that we consider here stability is achievable for large detunings for the trapping laser field and small detunings of the cooling field. It is therefore not always possible to stay within the window of detunings $|\Delta| \leqslant \sqrt{3} \gamma / 2$ where the 2MC dynamics are monostable, and bistability inevitably becomes an issue.

To investigate the effect of noise on the steady-state values Eq. (4) we consider each operator in Eq. (3) as the sum of its corresponding $c$-number mean value from Eq. (44) and a small fluctuation around that value. For example, $a=a_{s}+\delta a$. Using these definitions in Eq. (3), eliminating the steady-state conditions and linearizing the remaining equations in the fluctuations, we can write compactly

$$
\dot{\bar{u}}(t)=A \bar{u}(t)+\bar{n}(t) .
$$

Here the input noise vector is given by $\bar{n}(t)=$ $\left(\sqrt{\gamma} X_{a}^{\text {in }}, \sqrt{\gamma} Y_{a}^{\text {in }}, \sqrt{\gamma} X_{b}^{\text {in }}, \sqrt{\gamma} Y_{b}^{\text {in }}, 0, \delta \epsilon^{\text {in }}\right)$ and the vector of fluctuations in the dynamical variables by $\bar{u}(t)=$ $\left(\delta X_{a}, \delta Y_{a}, \delta X_{b}, \delta Y_{b}, \delta Q, \delta P\right)$, where we have redefined the field fluctuations as $\delta X_{a}=\left(\delta a+\delta a^{\dagger}\right) / \sqrt{2}$ and $\delta Y_{a}=$ $\left(\delta a-\delta a^{\dagger}\right) / i \sqrt{2}$, for example. Further,

$$
A=\left(\begin{array}{cccccc}
-\frac{\gamma}{2} & \Delta & 0 & 0 & 0 & 0 \\
-\Delta & -\frac{\gamma}{2} & 0 & 0 & \sqrt{2} \hbar \xi f_{s} & 0 \\
0 & 0 & -\frac{\gamma}{2} & \Delta & 0 & 0 \\
0 & 0 & -\Delta & -\frac{\gamma}{2} & -\sqrt{2} \hbar \xi f_{s} & 0 \\
0 & 0 & 0 & 0 & 0 & \frac{1}{M} \\
\sqrt{2} \hbar \xi f_{s} & 0 & -\sqrt{2} \hbar \xi f_{s} & 0 & -M \Omega_{M}^{2} & -\frac{T_{M}}{2 M}
\end{array}\right) .
$$

The solutions to Eq.(5) are stable when none of the eigenvalues of the matrix $A$ has a positive real part. This condition can be formalized using the Routh-Hurwitz criterion [27]; the corresponding inequalities are too involved to be reproduced here, but we have verified that they hold for the parameter values considered in this Letter.

In order to solve Eq.(5) we Fourier transform it into a set of algebraic equations. We then find the mechanical susceptibility $\chi(\omega)$ of the middle mirror by solving for $\delta Q(\omega)=\chi(\omega) F_{T}(\omega)$ [16], where $F_{T}$ is the total force on the middle mirror and includes the Brownian and radiation forces. The susceptibility has the form of a Lorentzian $\chi^{-1}(\omega)=M\left(\Omega_{\text {eff }}^{2}-\omega^{2}\right)-i \Gamma_{\text {eff }} \omega[16$, where the effective oscillation frequency and damping for the mirror are given by

$$
\begin{aligned}
& \Omega_{\mathrm{eff}}^{2}=\Omega_{M}^{2}-\frac{4 \xi \gamma P_{\mathrm{in}}}{M L} \frac{\Delta}{\Delta^{2}+\frac{\gamma^{2}}{4}}\left[\frac{\Delta^{2}-\omega^{2}+\frac{\gamma^{2}}{4}}{\left(\Delta^{2}-\omega^{2}+\frac{\gamma^{2}}{4}\right)^{2}+\gamma^{2} \omega^{2}}\right], \\
& \Gamma_{\mathrm{eff}}=\frac{\Gamma_{M}}{2}+\frac{4 \xi \gamma P_{\text {in }}}{M L} \frac{\Delta}{\Delta^{2}+\frac{\gamma^{2}}{4}}\left[\frac{\gamma}{\left(\Delta^{2}-\omega^{2}+\frac{\gamma^{2}}{4}\right)^{2}+\gamma^{2} \omega^{2}}\right] .
\end{aligned}
$$

In the static limit $(\omega=0) \Omega_{\text {eff }}$ reduces to the classical expression for the frequency of the mirror motion in the lowest minima of the potential shown in Fig $1(d)$.

In order to carry out a comparison we calculate the corresponding effective quantities $\omega_{\text {eff }}, \gamma_{\text {eff }}$ for the $2 \mathrm{MC}$ in a similar way and with identical parameters, but pumping with $2 P_{\text {in }}[\operatorname{Fig} \mathbb{1}(\mathrm{a})]$. (We preserve the notation for the fixed mirror and the optical fields, but use lower case for the movable mirror variables.) In this paper we restrict ourselves to comparing the frequencies $\omega_{\text {eff }}$ and $\Omega_{\text {eff }}$ for brevity.

The expressions for $\omega_{\text {eff }}$ and $\gamma_{\text {eff }}$ turn out to be identical to Eq. (77) except for the replacement of $\Delta$ by the effective detuning

$$
\Delta^{\prime}=\Delta-\xi q_{s}
$$

Here $\xi q_{s}$ is the radiation pressure induced detuning due to the non-zero equilibrium position for the mirror, $q_{s}$ [Fig1(a)]. This displacement is determined from the steady-state balance of the radiation and spring forces on the mirror

$$
\frac{2 \gamma P_{\text {in }} / L}{\left(\frac{\gamma}{2}\right)^{2}+\left(\Delta-\xi q_{s}\right)^{2}}-m \omega_{m}^{2} q_{s}=0,
$$

which is a cubic in $q_{s}$. Below the onset of bistability Eq. (9) has a single real root that is always positive and grows with input power $\left(q_{s} \sim P_{\text {in }}^{1 / 3}\right)$. For high $P_{\text {in }}$ the 
vibrational properties of the mirror are dominated by the optical fields, that is, the contributions due to $\Omega_{M}$ and $\Gamma_{M}$ in Eq.(7) are negligible compared to the remaining terms and similarly for $\omega_{\text {eff }}, \gamma_{\text {eff }}$.

Since the spring is soft we examine the frequency ratio at $(\omega \ll \gamma / 2)$ [24]. This limit essentially yields the classical potentials derived earlier [20] and shown in Figs [1(b) and (d). The frequency ratio

$$
\frac{\Omega_{\mathrm{eff}}}{\omega_{\mathrm{eff}}} \sim\left(\frac{\Delta}{\Delta^{\prime}}\right)^{1 / 2}\left(\frac{\Delta^{\prime 2}+\gamma^{2} / 4}{\Delta^{2}+\gamma^{2} / 4}\right)
$$

implies a very significant improvement in trapping in the $3 \mathrm{MC}$ as compared to the $2 \mathrm{MC}$ since $|\Delta|<\left|\Delta^{\prime}\right|$ recall that the number of quanta $n$ scales as $\Omega_{\text {eff }}^{-3}$, see Eq. (11). For $M=1 \mathrm{mg}, \Omega_{M}=2 \pi \times 100 \mathrm{~Hz}, L=2.5 \mathrm{~cm}$, $\gamma=10 \mathrm{MHz}, \lambda=1064 \mathrm{~nm}, P_{\text {in }}=1 \mathrm{~mW}$ and $\Delta=-\gamma / 2$, $\Delta^{\prime} \sim-4.5 \gamma$ and $\left(\Omega_{\mathrm{eff}} / \omega_{\mathrm{eff}}\right) \sim 10$. The actual frequencies are $1.6 \Omega_{M}$ and $18 \Omega_{M}$ for the $2 \mathrm{MC}$ and $3 \mathrm{MC}$ configurations, respectively.

It is clear that accounting for $\left(\Omega_{\text {eff }} / \omega_{\text {eff }}\right)$ in Eq. (10) and assuming similar cold damping performance, we can achieve numbers of quanta three orders of magnitude smaller in the $3 \mathrm{MC}$ than in the $2 \mathrm{MC}$ configuration. Specifically, for our cavity parameters and the length $L$ shortened to $L=1 \mathrm{~cm}$, trapping light at $P_{\mathrm{in}}^{t}=400 \mathrm{~mW}$, $\Delta^{t}=-2.5 \gamma$ and cooling light at $P_{\text {in }}^{c}=5 \mathrm{~mW}, \Delta^{c}=\gamma / 2$, it is possible to achieve $n<1$ when starting from room temperature, that is, the mirror can be cooled to its ground state of motion.

Having demonstrated the advantages of the $3 \mathrm{MC}$ configuration, we now discuss limitations and ramifications of our proposal. It is particularly relevant to consider here the role of feedback as it can influence the mirror stiffness. In the $2 \mathrm{MC}$ case, if the laser is locked to the cavity [3], or if a transducer is used to control the 'fixed' mirror and keep the cavity length constant [1], the movable mirror feels the spring force from both sides, but the radiation pressure force only from one side yielding the asymmetric potential of Fig प(b).

However if the transducer (such as a piezoelectric crystal or magnetic coil) is mounted on the movable mirror 24], then feedback governing the length of the cavity provides a force equal and opposite to the radiation pressure force in the cavity (within the bandwidth of the loop), yielding a potential similar to the $3 \mathrm{MC}$ case shown in Fig1(d). But such a force brings with it classical noise, since it essentially corresponds to (one-sided) stiffening of the mechanical connection of the mirror. Moreover, with nanomechanical oscillators being used in many experiments $[1,2$, 3, 4 4 the possibility of using purely optical control seems more attractive for miniaturization as well as scaling. The $3 \mathrm{MC}$ configuration that we propose is in a sense a form of feedback where the noise contribution is quantum mechanical, as classical noise can be suppressed in the laser light. The same effect could be achieved with an independent laser beam used from the right on the $2 \mathrm{MC}$, but it would require impracticably high powers. The addition of an extra cavity allows for lower laser power to build up to the same effect. With the higher optical stiffness that can be achieved with the $3 \mathrm{MC}$ the role of classical noise is therefore reduced further.

In conclusion we have proposed a laser cooling and trapping geometry that provides stiffer traps, removes bistability partially, and isolates the mirror further from classical mechanical noise. We believe that it offers a promising experimental route for confining and cooling a macroscopic mirror near its quantum mechanical ground state than heretofore proposed or achieved.

This work is supported in part by the US Office of Naval Research, by the National Science Foundation, by the US Army Research Office, by the Joint Services Optics Program, and by the National Aeronautics and Space Administration. We would like to thank H. Uys for stimulating discussions.
[1] S. Gigan et al., Nature 444, 67 (2006).

[2] D. Kleckner and D. Bouwmeester, Nature 444, 75 (2006).

[3] O. Arcizet et al., Nature 444, 71 (2006).

[4] A. Schliesser et al., Phys. Rev. Lett. 97, 243905 (2006).

[5] A. D. Armour, M. P. Blencowe, and K. C. Schwab, Phys. Rev. Lett. 88, 148301 (2002); M. D. LaHaye et al., Science 304, 74 (2004); A. Naik et al., Nature 443, 193 (2006).

[6] A. J. Leggett, J. Phys. Condens. Matter 14, R415 (2002).

[7] W. Marshall et al., Phys. Rev. Lett. 91, 130401 (2003).

[8] S. Mancini et al., Phys. Rev. Lett. 88, 120401 (2002).

[9] P. Treutlein et al., arXiv:quant-ph/0703199v1 (2007).

[10] C. M. Caves, Phys. Rev. Lett. 45, 75 (1980).

[11] D. Vitali et al., Phys. Rev. Lett. 98, 030405 (2007).

[12] J.-M. Courty, A. Heidmann, and M. Pinard, Phys. Rev. Lett. 90, 083601 (2003).

[13] G. J. Milburn, K. Jacobs and D. F. Walls, Phys. Rev. A 50, 5256 (1994).
[14] A. Dorsel et al., Phys. Rev. Lett. 51, 1550 (1983).

[15] V. B. Braginsky and S. P. Vyatchanin, Phys. Lett. A 293, 228 (2002).

[16] P. F. Cohadon, A. Heidmann and M. Pinard, Phys. Rev. Lett. 83, 3174 (1999).

[17] C. H. Metzger and K. Karrai, Nature 432, 1002 (2004).

[18] M. Pinard et al., Phys. Rev. A 63, 013808 (2000).

[19] M. Poggio et al., arXiv:cond-mat/0702446v1 (2007).

[20] P. Meystre et al., J. Opt. Soc. Am. B 11, 1830 (1985); J. D. McCullen, P. Meystre, and E. M. Wright, Optics Letters 9, 193 (1984).

[21] F. Ya. Khalili, Phys. Lett. A 288, 251 (2001).

[22] M. Vogel et al., App. Phys. Lett. 83, 1337 (2003).

[23] B. S. Sheard et al., Phys. Rev. A 69, 051801(R) (2004).

[24] T. Corbitt et al., Phys. Rev. Lett 98, 150802 (2007).

[25] C. Gardiner, Quantum Noise, Springer-Verlag, Berlin, (1991).

[26] S. Mancini and P. Tombesi, Phys. Rev. A 49, 4055 
(1994).

[27] E. X. DeJesus and C. Kaufman, Phys. Rev. A 35, 5288

(1987) 